\title{
Fenótipo ampliado do autismo e habilidades pragmáticas em pais e mães de crianças com e sem transtorno do espectro autista
}

\author{
fenotipo ampliado del autismo y habilidades pragmáticas en padres y madres de \\ niños con y sin trastorno del espectro autista \\ Broader Autism Phenotype and Pragmatic fbilities in Parents of Children with and \\ without futistic Spectrum Disorder
}

\author{
Renata Giuliani Endres* \\ Juliana Burges Sbicigo \\ Jerusa Fumagalli de Salles \\ Cleonice Alves Bosa \\ Universidade Federal do Rio Grande do Sul
}

Doi: https://doi.org/10.12804/revistas.urosario.edu.co/ap1/a.7855

\begin{abstract}
Resumo
$\bar{O}$ fenótipo ampliado do autismo (FAA) tem sido definido como traços subclínicos que configuram a expressão fenotípica de uma suscetibilidade genética para o desenvolvimento do Transtorno do Espectro Autista (TEA). A literatura tem apontado para a relação entre habilidades comunicativo-pragmáticas e autismo. No entanto, há controvérsia quanto à possibilidade dessas habilidades constituírem uma especificidade do FAA. Ainda, pesquisas com parentes de primeiro grau de pessoas com TEA ainda não obtiveram sucesso em estabelecer marcadores definitivos. Assim, este estudo comparou o desempenho de pais e mães de crianças com TEA $(n=26)$ e sem TEA
\end{abstract}

( $n=26)$ em tarefas de habilidades comunicativo-pragmáticas e indicadores de FAA. Foram aplicados o Broader Autism Phenotype Questionnaire (BAPQ) e subtestes da Bateria Montreal de Avaliação da Comunicação (Bateria MAC), além da Escala de Inteligência Wechsler Abreviada (WASI). Os resultados indicaram que os pais de crianças com TEA apresentaram maior dificuldade em linguagem pragmática do $\mathrm{BAPQ}$, ainda que não tenham fechado critério para FAA, e nas habilidades comunicativo-pragmáticas de discurso conversacional, prosódia emocional, interpretação de metáforas e de atos de fala indiretos. Foi também verificado que o grupo clínico apresentou menor escore em QI verbal em relação ao

Dirigir correspondência à Renata Giuliani Endres, Universidade Federal do Rio Grande do Sul. Correio eletrônico: rgendres01@ gmail.com

Renata Giuliani Endres ORCID ID: http://orcid.org/0000-0003-2090-8211

Para citar este artigo: Endres, R. G., Sbicigo, J. B., Salles, J. F., \& Bosa, C. A. (2020). Fenótipo ampliado do autismo e habilidades pragmáticas em pais e mães de crianças com e sem transtorno do espectro autista. Avances en Psicología Latinoamericana, 38(2), 1-16. https://doi.org/10.12804/revistas.urosario.edu.co/apl/a.7855 
grupo comparativo. Conclui-se que maiores dificuldades em habilidades cognitivo-pragmáticas podem ser um aspecto central e provavelmente específico do FAA em familiares de crianças com TEA, assim como dificuldades em aspectos estruturais da linguagem, como inteligência verbal. Estes resultados têm implicações para a compreensão da possível influência de fatores sociocognitivos familiares na gênese do autismo.

Palavras-chave: fenótipo ampliado do autismo; habilidades comunicativo-pragmáticas; genitores; quociente de inteligência.

\section{Resumen}

El fenotipo ampliado del autismo (FAA) se ha definido como trazos subclínicos que configuran la expresión fenotípica de una susceptibilidad genética para el desarrollo del Trastorno del Espectro Autista (TEA). La literatura ha apuntado hacia la relación entre habilidades comunicativo-pragmáticas y autismo. Sin embargo, hay controversia en cuanto a la posibilidad de que esas habilidades constituyan una especificidad del FAA. Así mismo, investigaciones con parientes de primer grado de personas con TEA han fallado en establecer marcadores definitivos. Este estudio comparó el desempeño de padres y madres de niños con TEA $(n=26)$ y $\sin$ TEA $(n=26)$ en tareas de habilidades comunicativo-pragmáticas e indicadores de FAA. Fueron aplicados el Broader Autism Phenotype Questionnaire (BAPQ) y subpruebas de la Batería Montreal de evaluación de la Comunicación (Batería MAC), además de la Escala de Inteligencia Wechsler Abreviada (WASI). Los resultados indicaron que los padres de niños con TEA presentaron mayor dificultad en lenguaje pragmático del BAPQ, aunque no hayan cumplido el criterio para FAA, y en las habilidades comunicativo-pragmáticas de discurso conversacional, prosodia emocional, interpretación de metáforas y de actos de habla indirectos. También se verificó que el grupo clínico presentó menor puntaje en CI verbal en relación con el grupo comparativo. Se concluyó que mayores dificultades en habilidades cognitivo-pragmáticas pueden ser un aspecto central y probablemente específico del FAA en familiares de niños con TEA, así como dificultades en aspectos estructurales del lenguaje, como inteligencia verbal. Estos resultados tienen implicaciones para comprender la posible influencia de factores sociocognitivos familiares en la génesis del autismo.

Palabras clave: fenótipo ampliado del autismo; habilidades comunicativo-pragmáticas; los padres; cociente intelectual.

\section{fibstract}

The broader autism phenotype (BAP) has been defined as subclinical traits that configure the phenotypic expression of genetic susceptibility to the development of Autistic Spectrum Disorder (ASD). The literature has pointed to the relationship between communicative-pragmatic skills and autism. However, there is controversy as to whether these skills constitute a specific feature of the BAP. Still, research with first-degree relatives of people with ASD has not yet been successful in establishing definitive markers. Thus, this study compared the performance of parents of children with $(n=26)$ and without ASD $(n=26)$ in communicative-pragmatic skills tasks and BAP indicators. The Broader Autism Phenotype Questionnaire (BAPQ) and subtests of the Montreal Communication Evaluation Battery (MAC Battery), and the Wechsler Abbreviated Intelligence Scale (WASI) were applied. The results indicated that parents of children with ASD had greater difficulty in the pragmatic language of the BAPQ, although they did not meet the criteria for BAP and in communicative-pragmatic skills of conversational speech, emotional prosody, metaphor interpretation, and indirect speech acts. The clinical group also presented a lower verbal IQ score compared to the nonclinical group. It is concluded that greater difficulties in cognitive-pragmatic skills may be a central and probably specific aspect of the BAP in family members of children with ASD, as well as difficulties in structural aspects of language, such as verbal intelligence. These results have implications for understanding the possible influence of familiar socio-cognitive factors on the genesis of autism.

Keywords: Broader autism phenotype; communicative-pragmatic skills; parents; intelligence quotient. 
O transtorno do espectro autista (TEA) consiste em um amplo conjunto de condições neuro desenvolvimentais, caracterizado por dificuldades significativas nos domínios sociocomunicativos e por um repertório restrito e repetitivo de comportamentos, interesses e atividades (American Psychiatric Association [APA], 2013). De acordo com os resultados de algumas pesquisas e com a quinta versão do Manual Diagnóstico e Estatístico de Transtornos Mentais (DSM-5), somam-se a estes comprometimentos as dificuldades no processamento sensorial, mais especificamente a ocorrência de organização perceptual atípica (Mottron et al., 2013; Van der Hallen, Evers, Brewaeys, Van den Noortgate \& Wagemans, 2015).

Estudos com pais e irmãos não afetados de indivíduos com TEA identificaram características de personalidade (ex.: introversão), de linguagem (ex.: pragmática) e a presença de comportamentos (ex.: rigidez) que refletem a expressão fenotípica de genes candidatos à suscetibilidade para desenvolver o TEA (Davidson et al., 2014; Rubenstein \& Chawla, 2018). No contexto brasileiro, os resultados de um dos poucos estudos existentes (Endres, Lampert, Schuch, Roman \& Bosa, 2015) demonstraram que genitores de crianças com TEA tendem a apresentar traços de personalidade que parecem corresponder às características comportamentais das áreas que compõem o transtorno (ex: tendência à rigidez e ao retraimento social), sugerindo a possível presença do fenótipo ampliado do autismo. Esse estudo investigou o perfil de personalidade de 20 pais e mães de crianças com o diagnóstico de TEA, através do delineamento de estudos de casos múltiplos, utilizando a Bateria Fatorial de Personalidade (BFP) e o Broad Autism Phenotype Questionnaire (BAPQ). Esses parentes, apesar de não preencherem os critérios para o diagnóstico clínico do transtorno, expressam traços de personalidade e sintomas subclínicos que vão de um nível mais leve a um nível moderado da condição (Dawson et al., 2002; Ingersoll, 2010), definidas como o fenótipo ampliado do autismo (FAA) (Piven, 2001).
Nessa linha de pesquisas, um estudo encontrou uma taxa de herdabilidade moderada (Hallmayer et al., 2011). No entanto, mais recentemente, Tick, Bolton, Happé, Rutter e Rijsdijk (2016) conduziram uma meta-análise, utilizando as bases Pubmed Central, Science Direct, Google Acadêmico e Web of Knowledge, para identificar os estudos com irmãos gêmeos de pessoas com TEA publicados até a data. Dos treze estudos identificados, sete foram incluídos na pesquisa, dentre eles uma amostra populacional de 6413 pares de gêmeos. Os resultados demonstraram que o TEA ocorre em decorrência de efeitos genéticos significativos. Sobre a discrepância entre os resultados das pesquisas, Waye e Cheng (2018) explicaram que isto se deve a mudanças encontradas ao longo dos anos quanto à etiologia dos diferentes tipos de autismo. Os autores concluíram que as causas da variabilidade de TEA parecem ser muito complexas e incluem fatores ambientais (ex.: medicamentos), genéticos e biológicos (ex.: comorbidades).

Entre as dimensões do FAA que têm sido foco de pesquisa está a área da linguagem. Algumas evidências sugerem que pais e irmãos de indivíduos com TEA apresentam maiores dificuldades na utilização da linguagem para comunicação com propósitos sociais (pragmática) se comparados a outros grupos clínicos (Flax et al., 2019; Sucksmith, Roth \& Hoekstra, 2011). Foram encontrados maiores níveis de dificuldades de linguagem em habilidades pragmáticas nos pais de crianças com autismo quando comparados a pais dos grupos controle, clínicos (e.g. transtorno específico de linguagem) e não clínicos, utilizando Autism-Spectrum Quotient (Ruta et al., 2012; Wheelwright, Auyeung, Allison \& Baron-Cohen, 2010; Whitehouse, Barry \& Bishop, 2007). Essa escala consiste em um questionário auto administrado para quantificar o número de traços autísticos que um indivíduo possui em cinco domínios (habilidade social, mudança de atenção, atenção aos detalhes, comunicação e imaginação). Losh et al. (2008) verificaram maior comprometimentos no uso da linguagem pragmática, prosódia e discurso 
gramatical em famílias com mais de uma criança com TEA que em famílias com apenas um filho com TEA ou famílias com filho com síndrome de Down, sugerindo que as dificuldades de linguagem possam ser geneticamente influenciadas.

No entanto, os achados sobre a linguagem parecem ser ainda inconsistentes, sobretudo em tarefas de fluência verbal que envolvem a produção de palavras de acordo com uma regra (letras para fluência fonêmica e categoria para fluência semântica), em um intervalo de tempo (Camodeca, Todd \& Hosack, 2018). Por exemplo, Camodeca e Voelker (2016) demonstraram um lento processamento controlado no grupo FAA, e que essas fraquezas eram preditivas de problemas no uso da linguagem pragmática no cotidiano. Contudo, Camodeca et al. (2018), ao replicarem os resultados encontrados por Camodeca e Voelker (2016), em 182 graduandos, utilizando o teste de Fluência Verbal do Sistema de Função Executiva Delis Kaplan (D-KEFS-VF) e o Questionário de Fenótipo Ampliado de Autismo (BAPQ) não encontram os mesmos resultados. O grupo FAA $(n=$ 31) não demonstrou dificuldades no processamento controlado, nem o desempenho nesta tarefa foi preditivo dos resultados na dimensão Linguagem Pragmática do BAPQ. Porém, tal como no estudo anterior, o processamento controlado não foi preditivo de habilidades de linguagem pragmática para indivíduos sem traços de TEA $(n=151)$. Os resultados revelaram que as habilidades de fluência verbal no FAA são semelhantes às do grupo comparativo, e que o processamento controlado não explica a variância no uso da linguagem pragmática no FAA.

As inconsistências nos achados também se revelam na aquisição, compreensão e articulação do discurso, em parentes de indivíduos com autismo. Em cerca de $22 \%$ de irmãos de crianças com autismo, entre dois e seis anos de idade, houve atraso de linguagem (Chuthapisith, Ruagdaraganon, Sombuntham \& Roongpraiwan, 2007). No estudo de Lee et al. (2019), com 37 indivíduos com TEA e 38 controles, 151 pais de indivíduos com TEA e 63 controles (pais), em tarefas que avaliaram a quali- dade da narrativa. Os grupos com TEA e pais/TEA demonstraram menor qualidade nesta habilidade do que os grupos comparativos, porém nas tarefas que foram menos estruturadas

Além de mostrar atraso no desenvolvimento da linguagem, alguns familiares de crianças com TEA também apresentaram dificuldades em tarefas que avaliavam os aspectos estruturais da linguagem (discurso, sintaxe, semântica e coerência), sugerindo marcadores de comprometimento hereditário destes aspectos (Lindgren et al., 2009). Em contraste, outros autores afirmam que as dificuldades de comunicação que caracterizam o FAA podem ser restritas ao domínio pragmático. Por exemplo, Whitehouse et al. (2007) relataram que pais de crianças com TEA apresentavam comprometimentos na linguagem pragmática, mas não em aspectos estruturais da linguagem. Esses achados foram replicados em um estudo maior que incluiu amostras no Reino Unido e nos EUA, no qual os autores confirmaram que a dificuldade de comunicação predominantemente encontrada em pais de crianças com TEA foi no engajamento social, isto é, no aspecto pragmático, e não na estrutura de linguagem (Whitehouse et al., 2010).

Estudos mais recentes apoiam a hipótese de que os déficits de comunicação social são parte do fenótipo herdável do TEA (Bernier, Gerdts, Munson, Dawson \& Estes, 2012; Gerdts, Bernier, Dawson \& Estes, 2013). Esses resultados também indicaram que os pais de famílias com mais de um filho com TEA demonstram um maior grau de características da condição quando comparados a pais das famílias com apenas um filho com o transtorno, particularmente em domínios sociais e de comunicação.

O estudo de Taylor et al. (2013) explorou a relação entre a presença de traços do FAA dos pais de crianças com TEA e dificuldades de comunicação em seus filhos. Os resultados dessa pesquisa mostraram que as crianças com pelo menos um dos genitores com FAA + tiveram escores mais baixos, tanto na subescala de linguagem estrutural quanto pragmática, quando comparados com crianças com 
pais com FAA-. Desse modo, é possível que exista uma interação gene-ambiente, em que as crianças com autismo que possuem pais com traços autísticos $\left(\mathrm{FAA}^{+}\right)$tenham tanto uma maior suscetibilidade genética para comprometimentos sociais de linguagem, quanto menor possibilidade de aprender aspectos importantes da comunicação através de seus pais, uma vez que os próprios podem apresentar dificuldades nos aspectos estruturais ou pragmáticos da linguagem (Taylor et al., 2013).

Por fim, outro domínio com achados contraditórios quanto à relação com os traços autísticos é o que trata dos aspectos estruturais da linguagem por meio da avaliação das habilidades intelectuais. Por exemplo, irmãos de crianças com TEA apresentaram QI verbal mais alto na comparação com irmãos de crianças com Síndrome de Down (Fombonne, Bolton, Prior, Jordan \& Rutter, 1997; Pilowsky, Yirmiya, Shalev \& Gross-Tsur, 2003), porém essa diferença não foi encontrada em outras pesquisas (ex.: Folstein et al., 1999; Pilowsky, Yirmiya, Gross-Tsur \& Shalev, 2007). Quanto aos pais de crianças com TEA, uma recente revisão sistemática (Ruparelia, Manji, Abubakar \& Newton, 2017) indicou que de 30 estudos avaliando QI verbal, apenas um mostrou diferenças significativas, com escore mais alto desses pais em comparação a pais de crianças com Síndrome de Down (Fombonne et al., 1997). Por outro lado, também foi observada uma tendência inversa, com os pais de crianças com TEA apresentando escore significativamente mais baixo em QI verbal do que pais de crianças com desenvolvimento típico (Smalley \& Asarnow, 1990). Em todos os estudos, os escores dos pais de crianças com TEA estiveram dentro da média. Assim, os resultados não são claros em relação ao papel do QI verbal no FAA (Ruparelia et al., 2017).

O objetivo desse estudo foi comparar indicadores do FAA e habilidades comunicativo-pragmáticas entre pais e mães de crianças com TEA e pais e mães de crianças com desenvolvimento típico. A principal hipótese foi que o grupo de pais e mães de crianças com autismo apresentariam mais indi- cadores de FAA do que os pais e mães de crianças com desenvolvimento típico, incluindo escores mais baixos nas dimensões que avaliam os aspectos comunicativos da linguagem. Considerando as inconsistências na literatura, examinou-se também eventuais diferenças entre os dois grupos em relação aos aspectos estruturais da linguagem por meio da avaliação do QI verbal (vocabulário).

\section{Método}

\section{Participantes}

O estudo teve delineamento de grupos contrastantes (Naschmias \& Naschmias, 1996), com 13 pais e 13 mães de crianças com diagnóstico de TEA (grupo TEA), e 13 pais e 13 mães de crianças com desenvolvimento típico (grupo DT), totalizando 52 participantes (26 em cada grupo). Os participantes do grupo comparativo foram equiparados aos do grupo TEA por sexo e média de idade dos genitores (tabela 1).

Os critérios de inclusão de pais e mães do grupo TEA foram: ser pais biológicos da criança com TEA; participação do casal na pesquisa; assinatura do Termo de Consentimento Livre e Esclarecido pelo casal (TCLE); ausência de deficiências físicas e/ou sensoriais dos pais (não corrigidas) e de qualquer doença que impedisse o processo de avaliação; e QI acima de 70 na Escala de Inteligência Weschler Abreviada (WASI). Para participação no grupo comparativo, foram utilizados os mesmos critérios descritos para o grupo clínico, além do critério de ausência de atrasos ou transtornos desenvolvimentais nos filhos, conforme o Questionário de Capacidades e Dificuldades (SDQ-POR) (Fleitlich, Cortázar \& Goodman, 2000).

A amostra do grupo TEA foi composta por pais e mães de crianças previamente avaliados no Centro Experimental de Avaliação Multidisciplinar em Autismo (CEMA-UFRGS), os quais compõem o banco de dados do serviço, e também em outros 
centros de atendimento à comunidade. Os filhos dos indivíduos que fazem parte deste estudo foram previamente diagnosticados por médicos especialistas e fecham critérios para o TEA, de acordo com a Classificação Internacional de Doenças e Problemas Relacionados à Saúde (CID 10). Os pais das crianças do grupo comparativo foram selecionados por meio da técnica de amostragem bola de neve. Para cada família do grupo TEA, contatou-se uma família para compor o grupo comparativo, equiparando os participantes de ambos os grupos por idade dos pais e das mães. Além de contato com famílias previamente avaliadas no CEMA-UFRGS, para fins de ampliação da amostra, estas famílias do grupo clínico indicaram casais com filhos diagnosticados com TEA, que igualmente preenchessem os critérios de inclusão na pesquisa. Da mesma forma, procedeu-se à seleção dos participantes do grupo comparativo.

\section{Instrumentos}

Dados Sociodemográficos: foram coletadas informações como sexo, idade, número de filhos e idade dos filhos. Foi também utilizado o Questionário Critério Brasil (Associação Brasileira de Empresas de Pesquisa [ABEP], 2015), um índice combinando escolaridade, posse de bens duráveis e renda familiar para a caracterização do nível socioeconômico (NSE). Nesse estudo, foi utilizado o escore total do questionário.

Self Report Questionnaire (SRQ-20) (Harding et al., 1980; Mari \& Williams, 1986): investiga transtornos mentais comuns. Os 20 itens são distribuídos de acordo com quatro grupos de sintomas (humor depressivo-ansioso; sintomas somáticos, decréscimo de energia vital e pensamentos depressivos). Sete ou mais respostas afirmativas ( sim) configuraram a presença de sofrimento mental.

$B A P Q$ (Hurley et al., 2007; Prata, Junior, Teodoro \& Rocha, 2019): avalia características específicas de personalidade e de linguagem postuladas como traços marcantes do FAA. Tais características incluem personalidade social, personalidade rígida e déficits pragmáticos da linguagem, em pais de indivíduos com TEA (Piven et al., 1997). As três dimensões do BAPQ, Interação Social, Linguagem Pragmática e Rigidez, fornecem índices quantitativos relevantes para os três domínios do TEA, presentes no DSM-IV (APA, 2013). Este instrumento foi submetido ao processo de adaptação (Cruz, 2012). De acordo com os parâmetros e diretrizes da Organização Mundial da Saúde (World Health Organization [WHO], 1993). Para evitar possíveis vieses, o protocolo de respostas entregue aos participantes foi denominado de "Questionário de Estilos de Personalidade e Preferências", ao invés de Questionário do FAA. As respostas aos 36 itens do BAPQ são registradas em uma escala Likert. A interpretação dos resultados se dá por meio de pontos de corte em cada uma das dimensões (Interação Social, $\mathrm{PC}=3.25$; Linguagem Pragmática, $\mathrm{PC}=2.75$, Rigidez, $\mathrm{PC}=3.50$ ) fornecendo, também, um escore total (das três dimensões; Escore Total, $\mathrm{PC}=3.50)$. Escores acima do ponto de corte configuram a presença de traços do FAA (Cruz, 2012; Hurley et al., 2007).

WASI (The Psychological Corporation, 1999; Trentini, Yates \& Heck, 2014): foram utilizados os subtestes de Vocabulário e Raciocínio Matricial.

Bateria Montreal de Avaliação da Comunicação - Bateria MAC (Ferré, Lamelin, Côté, Ska \& Joanette, 2011; Fonseca, Parente, Côte, Ska \& Joanette, 2008): quatro componentes do processamento comunicativo de ativação do hemisfério cerebral direito (HD): discursivo, pragmático-inferencial, léxico-semântico e prosódico. A bateria abrange aspectos linguísticos (gramaticais ou estruturais) e paralinguísticos (funcionais), ao invés de apenas investigarem os componentes formais ou estruturais da linguagem verbal (fonologia, morfossintaxe e semântica literal). É composta por 14 diferentes tarefas linguísticas das quais quatro, que são ligadas aos aspectos 
discursivo, pragmático-inferencial e pragmático da linguagem, foram aplicadas nesse estudo: 1) Discurso Conversacional; 2) Interpretação de Metáforas; 3) Interpretação de Atos de Fala Indiretos; e 4) Prosódia Emocional - Compreensão. $\mathrm{O}$ instrumento possui normas de acordo com a faixa etária. Existe um ponto de alerta para cada grupo normativo: três grupos etários (19-39 anos, 40-59 anos e 60-75 anos), subdivididos em dois grupos de escolaridade (dois a sete anos de estudo e oito anos ou mais).

\section{Procedimentos éticos e de coleta de dados}

O projeto foi aprovado pelo Comitê de Ética em Pesquisa do Instituto de Psicologia da Universidade Federal do Rio Grande do Sul. Pais e mães de crianças com e sem TEA foram convidados a fazer parte do estudo por contato telefônico e/ou e-mail. Posteriormente, foi agendado um encontro para avaliação em que os participantes entregaram um TCLE assinado. A administração dos instrumentos foi realizada pela pesquisadora, auxiliada por uma bolsista de iniciação científica devidamente treinada. O processo de avaliação foi realizado em um encontro individual, com duração média de cerca de 2 horas por participante, realizando-se um intervalo de aproximadamente 10 minutos para descanso, quando necessário. A administração dos instrumentos foi feita em ordem aleatória para controlar o efeito de interferência de um instrumento sobre o outro e o efeito de fadiga.

Inicialmente, foram contatados 15 casais do grupo clínico (grupo TEA). Foram excluídos do estudo dois casais desse grupo $(\mathrm{QI}<70 \mathrm{em}$ um dos participantes e a retirada do consentimento para participação na pesquisa por outro participante). Posteriormente, foram contatados 15 casais para compor o grupo comparativo (grupo DT). Desse grupo, dois casais foram excluídos da amostra (em ambos os casos por desistência de um dos participantes).

\section{Análise de dados}

Foram calculadas as estatísticas descritivas (média, desvio padrão, mediana, quartil, frequência e porcentagem) das variáveis. Como os dados não apresentaram distribuição normal, foram utilizados os testes de correlação de Spearman e $U$ de Mann-Whitney. Foi aplicada a correção pela taxa de falsa descoberta (false discovery rate (FDR) às múltiplas testagens (Benjamini \& Hochberg, 1995). Foram calculados os tamanhos de efeito para testes não paramétricos, utilizando a equação $r=z / \sqrt{N}$. $\mathrm{Na}$ fórmula, $\mathrm{z}$ é a distribuição $\mathrm{z}$ e $\mathrm{N}$ é o tamanho da amostra. A fim de comparar esse tamanho de efeito com outros tamanhos de efeito (por exemplo $d$ de Cohen), a fórmula: $d=2 r / \sqrt{ }(1-r 2)$ foi usada (Fritz, Morris \& Richler, 2012). Os tamanhos de efeito foram classificados como pequeno (0.2), moderado (0.5) e grande (0.8) de acordo com Cohen (1988). O nível de significância adotado foi de $5 \%$.

\section{Resultados}

\section{Análises preliminares}

As características sociodemográficas e de saúde mental dos pais do grupo TEA e grupo DT são apresentadas na tabela 1. Os grupos não diferiram $(p>$ $0.05)$ quanto à idade, idade dos filhos e número de filhos por casal. No grupo TEA, a maioria, isto é, $38 \%$ possuíam ensino médio completo, enquanto no grupo comparativo, a maior parte dos participantes (57\%) concluíram uma pós-graduação. Quanto à renda, $34 \%$ contavam com uma renda de mais de 16 salários mínimos no grupo TEA, enquanto no grupo DT, $62 \%$. No escore total do questionário ABEP (Critério Brasil), verificou-se que o grupo TEA apresentou menor NSE na comparação com o grupo DT. Como o NSE não foi correlacionado com os domínios cognitivo-linguísticos dentro de cada grupo, não foi necessário realizar análise de covariância nas análises posteriores (Field, 2018; Quade, 1967). 
Ainda quanto aos dados descritivos, todos eram casados no grupo DT, enquanto no grupo TEA, $80 \%$. Em relação à saúde mental, dos 26 participantes do grupo clínico, cinco (uma mulher e quatro homens, $19.23 \%$ ) apresentaram um escore que denota sofrimento mental no SRQ-20 (acima do ponto de corte $>$ 7, conforme Mari \& Williams, 1986). Os que apresentaram sofrimento mental (no grupo TEA) estavam em acompanhamento psicoterápico no momento da realização da pesquisa. Nenhum participante do grupo comparativo apresentou indícios de sofrimento mental, de acordo com o SRQ-20.

Tabela 1.

Caracterização Sociodemográfica e de Saúde Mental dos Participantes por Grupo

\begin{tabular}{|c|c|c|c|}
\hline & $\begin{array}{l}\text { Grupo TEA }(n=26) \\
M(D P) / f(\%)\end{array}$ & $\begin{array}{l}\text { Grupo DT }(n=26) \\
\qquad M(D P) / f(\%)\end{array}$ & $U$ \\
\hline Idade (anos) & $40.23(7.25)$ & $37.62(2.87)$ & 290.00 \\
\hline Idade dos filhos (meses) & $98.5(58.52)$ & $52.67(20.14)$ & 45.00 \\
\hline Número de filhos & $1.38(0.63)$ & $1.42(0.50)$ & 310.00 \\
\hline \multicolumn{4}{|l|}{ Escolaridade } \\
\hline Pós-graduação & $7(26.90)$ & $15(57.70)$ & - \\
\hline Superior completo & $7(26.90)$ & $9(34.60)$ & - \\
\hline Médio completo & $10(38.50)$ & $2(7.70)$ & - \\
\hline Fundamental compl. & $1(3.85)$ & & - \\
\hline Fundamental incompl. & $1(3.85)$ & & - \\
\hline \multicolumn{4}{|l|}{ Renda Familiar } \\
\hline Até 1 salário & $1(3.85)$ & & - \\
\hline De 1 até 2 salários & $2(7.70)$ & & - \\
\hline De 2 até 4 salários & $3(11.55)$ & $1(4.15)$ & - \\
\hline De 4 até 7 salários & $5(19.25)$ & $3(12.50)$ & - \\
\hline De 7 até 11 salários & $2(7.70)$ & $6(25.00)$ & - \\
\hline De 11 até 16 salários & $2(7.70)$ & $1(4.15)$ & - \\
\hline Mais de 16 salários & $9(34.60)$ & $15(62.50)$ & - \\
\hline \multicolumn{4}{|l|}{ Nível Socioeconômico } \\
\hline ABEP Total & $38.77(7.60)$ & $48.73(6.45)$ & $98.50 *$ \\
\hline \multicolumn{4}{|l|}{ Status Conjugal } \\
\hline Casados & $24(92.30)$ & $26(100)$ & - \\
\hline Divorciados & $2(7.70)$ & $0(0)$ & - \\
\hline \multicolumn{4}{|l|}{ Saúde Mental (SRQ-20) } \\
\hline $\begin{array}{l}\text { Sem sofrimento mental (Escore } \\
\text { Total }<7 \text { pontos) }\end{array}$ & $21(80.75)$ & $26(100)$ & - \\
\hline $\begin{array}{c}\text { Com sofrimento mental (Escore } \\
\text { Total }>7 \text { pontos) }\end{array}$ & $5(19.25)$ & & - \\
\hline
\end{tabular}

Nota: $* p<.0$ 


\section{Diferenças entre os Grupos}

As análises de diferenças entre os grupos quanto às características do FAA (instrumento BAPQ) são apresentadas na tabela 2. Verificou-se que tanto no grupo de pais TEA quanto DT os escores foram abaixo do ponto de corte nas três dimensões do BAPQ (interação social, linguagem pragmática e rigidez), significando ausência de dificuldades nas respectivas áreas. Além disso, os grupos também diferiram no Escore Total do BAPQ e mais marca- damente na dimensão de Linguagem Pragmática, com o grupo TEA apresentando desempenho inferior em comparação ao grupo DT $(p<0.05)$. O tamanho de efeito das diferenças entre os grupos foi de magnitude moderada.

Quanto aos indicadores de inteligência (QI), houve diferença significativa entre os grupos de pais TEA e DT nos escores de QI total e de QI verbal. O desempenho (medianas) de QI total e verbal dos participantes do grupo TEA foi inferior em relação às do grupo DT, com tamanho de efeito moderado (tabela 3 ).

Tabela 2.

Desempenho no Instrumento BAPQ nos Grupos Clínico e Comparativo

\begin{tabular}{|c|c|c|c|c|c|c|c|c|c|c|c|c|c|}
\hline \multirow[t]{2}{*}{ Dimensões } & \multirow[t]{2}{*}{$\begin{array}{l}\text { Pontos } \\
\text { de corte }\end{array}$} & \multicolumn{5}{|c|}{$\begin{array}{l}\text { Grupo TEA } \\
(n=26)\end{array}$} & \multicolumn{5}{|c|}{$\begin{array}{c}\text { Grupo DT } \\
(n=26)\end{array}$} & \multirow[b]{2}{*}{$U$} & \multirow[b]{2}{*}{$d$} \\
\hline & & $\begin{array}{c}M \\
(D P) \\
\end{array}$ & $M d n$ & IIQ & $\begin{array}{l}\text { Min; } \\
\text { Max }\end{array}$ & $S E$ & $\begin{array}{c}M \\
(D P) \\
\end{array}$ & $M d n$ & IIQ & $\begin{array}{l}\text { Min; } \\
\text { Max }\end{array}$ & $S E$ & & \\
\hline $\begin{array}{l}\text { Interação } \\
\text { Social }\end{array}$ & 3.25 & $\begin{array}{c}2.56 \\
(0.17) \\
\end{array}$ & 2.45 & 0.85 & $\begin{array}{l}1.41 ; \\
5.08 \\
\end{array}$ & 0.18 & $\begin{array}{c}2.29 \\
(0.74) \\
\end{array}$ & 2.20 & 0.95 & $\begin{array}{l}1.75 \\
2.20 \\
\end{array}$ & 0.14 & 284.50 & 0.28 \\
\hline $\begin{array}{l}\text { Linguagem } \\
\text { Pragmática }\end{array}$ & 2.75 & $\begin{array}{c}2.58 \\
(0.65) \\
\end{array}$ & 2.41 & 0.88 & $\begin{array}{l}1.61 ; \\
3.91\end{array}$ & 0.13 & $\begin{array}{c}2.14 \\
(0.55) \\
\end{array}$ & 2.16 & 0.67 & $\begin{array}{l}1.70 \\
2.37\end{array}$ & 0.11 & $210.50 * \dagger$ & 0.68 \\
\hline Rigidez & 3.50 & $\begin{array}{c}3.05 \\
(0.90) \\
\end{array}$ & 2.91 & 1.34 & $\begin{array}{l}1.75 \\
5.41 \\
\end{array}$ & 0.17 & $\begin{array}{c}2.76 \\
(0.72) \\
\end{array}$ & 2.62 & 0.73 & $\begin{array}{l}2.31 \\
3.04\end{array}$ & 0.14 & 269.00 & 0.35 \\
\hline $\begin{array}{l}\text { Escore } \\
\text { Total BAPQ } \\
\end{array}$ & 3.15 & $\begin{array}{c}2.73 \\
(0.66) \\
\end{array}$ & 2.61 & 0.82 & $\begin{array}{l}1.68 ; \\
4.66\end{array}$ & 0.13 & $\begin{array}{c}2.40 \\
(0.10) \\
\end{array}$ & 2.34 & 0.60 & $\begin{array}{l}2.01 \\
2.61 \\
\end{array}$ & 0.03 & $223.00 * \dagger$ & 0.61 \\
\hline
\end{tabular}

Nota. $. M d n=$ mediana; IIQ = intervalo interquartil; $S E$ = erro padrão; $U=$ Teste de Mann-Whitney

$* p<.05$

$\dagger p$-valores corrigidos pela taxa de falsa descoberta (false discovery rate - FDT)

Tabela 3.

Desempenho no Instrumento WASI nos Grupos Clínico e Comparativo

\begin{tabular}{|c|c|c|c|c|c|c|c|c|c|c|c|c|}
\hline & & $\begin{array}{l}\text { Grupo } \\
\quad(n=\end{array}$ & $\begin{array}{l}\text { O TEA } \\
26)\end{array}$ & & & & $\begin{array}{l}\text { Grup } \\
(n=\end{array}$ & $\begin{array}{l}\text { o DT } \\
26)\end{array}$ & & & & \\
\hline & $\begin{array}{c}M \\
(D P)\end{array}$ & $M d n$ & IIQ & $\begin{array}{l}\text { Min; } \\
\operatorname{Max}\end{array}$ & $S E$ & $M(D P)$ & $M d n$ & IIQ & $\begin{array}{l}\text { Min; } \\
\operatorname{Max}\end{array}$ & $S E$ & $U$ & $d$ \\
\hline QI Verbal & $54.57(8.62)$ & 57.00 & 13.00 & $\begin{array}{c}29.00 \\
66.00 \\
\end{array}$ & 1.69 & $\begin{array}{l}59.80 \\
(5.26) \\
\end{array}$ & 59.50 & 6.75 & $\begin{array}{c}49.00: \\
67.00 \\
\end{array}$ & 1.03 & $207.50 * \dagger$ & 0.70 \\
\hline QI Execução & $55.84(6.74)$ & 56.00 & 11.00 & $\begin{array}{c}44.00 \\
69.00\end{array}$ & 1.32 & $\begin{array}{l}58.11 \\
(5.68)\end{array}$ & 57.00 & 8.00 & $\begin{array}{l}47.00 \\
69.00\end{array}$ & 1.11 & 268.00 & 0.37 \\
\hline QI Total & $109.23(12.11)$ & 109.00 & 15.25 & $\begin{array}{l}80.00 \\
134.00\end{array}$ & 2.37 & $\begin{array}{c}116.50 \\
(8.9)\end{array}$ & 116.50 & 10.00 & 98.00 & 1.74 & $202.50 * \dagger$ & 0.72 \\
\hline
\end{tabular}

Nota. Mnd = mediana; IIQ = intervalo interquartil; $S E$ = erro padrão; $U=$ Teste de Mann-Whitney

${ }^{*} p<.05$

$\dagger p$-valores corrigidos pela taxa de falsa descoberta (false discovery rate - FDT) 
Ampliando-se as análises no campo da linguagem, examinou-se o desempenho dos dois grupos de pais em relação às habilidades comunicativo-pragmáticas na Bateria MAC. Os grupos de pais de crianças com TEA e sem TEA diferiram nas seguintes tarefas (tabela 4): (a) Discurso conversacional; (b) Interpretação de Metáforas - Explicações; Interpretação de Metáforas Novas - Explicações; Interpre- tação de Metáforas Idiomáticas - Explicações; (c) Interpretação de Atos de Fala Indiretos: Situações Diretas - Alternativas e (d) Prosódia Emocional - Compreensão. Pais e mães de crianças com desenvolvimento típico obtiveram escores mais altos nesses domínios $(p<0.05)$, com tamanho de efeito grande em Discurso Conversacional, e moderado nas demais habilidades.

Tabela 4.

Desempenho em Habilidades Comunicativo-Pragmáticas (Bateria MAC) por Grupo

\begin{tabular}{|c|c|c|c|c|c|c|c|c|c|c|c|c|}
\hline & \multicolumn{5}{|c|}{$\begin{array}{l}\text { Grupo TEA } \\
\qquad(n=26)\end{array}$} & \multicolumn{5}{|c|}{$\begin{array}{l}\text { Grupo DT } \\
(n=26)\end{array}$} & \multirow[b]{2}{*}{$U$} & \multirow[b]{2}{*}{$d$} \\
\hline & $\mathrm{M}(\mathrm{DP})$ & $M d n$ & IIQ & $\begin{array}{l}\text { Min; } \\
\text { Max }\end{array}$ & $S E$ & $\begin{array}{c}\mathrm{M} \\
(\mathrm{DP})\end{array}$ & $M d n$ & IIQ & $\begin{array}{l}\text { Min; } \\
\text { Max }\end{array}$ & $S E$ & & \\
\hline $\begin{array}{l}\text { Discurso } \\
\text { conversacional }\end{array}$ & $\begin{array}{l}29.31 \\
(3.51)\end{array}$ & 30 & 3.00 & $\begin{array}{l}20 \\
34\end{array}$ & 0.7 & $\begin{array}{l}32.88 \\
(2.08)\end{array}$ & 33 & 2.00 & $28 ; 40$ & 0.4 & $118.50^{* * \dagger}$ & 1.35 \\
\hline
\end{tabular}

Interpretação de

metáforas

Escore total de interpretação de metáforas 34.65

Explicações

$(4.26)$

$35 \quad 7.00 \quad 23 ; 40 \quad 0.8$

37.23

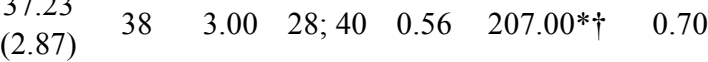

Escore total de metáforas novas -

Explicações

\subsection{0}

(2.75)

$\begin{array}{llll}17,5 & 3.00 & 8 ; 20 & 0.5\end{array}$

18.50

(1.50)

19

1.25

$5 ; 20 \quad 0.29 \quad 225.00 * \dagger$

0.61

Escore total de metáforas idiomáticas -

Explicações

$\begin{array}{lllll}17.57 & 18 & 3.00 & 12 ; 20 & 0.4\end{array}$

18.73

(1.82)

$19,5 \quad 2.25 \quad 13 ; 20 \quad 0.35 \quad 207.00 * \dagger$

0.72

\section{Interpretação de Atos \\ de Fala Indiretos}

\begin{tabular}{|c|c|c|c|c|c|c|c|c|c|c|c|c|}
\hline $\begin{array}{l}\text { Escore total de } \\
\text { situações diretas- } \\
\text { Alternativas }\end{array}$ & $\begin{array}{c}9.38 \\
(1.02)\end{array}$ & 10 & 1.00 & $6 ; 10$ & 0.2 & $\begin{array}{l}9.92 \\
(0.27)\end{array}$ & 10 & 0.00 & $9 ; 10$ & 0.05 & $231.00 * \dagger$ & 0.77 \\
\hline $\begin{array}{l}\text { Prosódia Emocional - } \\
\text { Compreensão }\end{array}$ & $\begin{array}{l}10.88 \\
(1.47)\end{array}$ & 11 & 1.25 & $6 ; 12$ & 0.3 & $\begin{array}{l}11.65 \\
(0.62)\end{array}$ & 12 & 1.00 & $10 ; 12$ & 0.12 & $214.00 * \dagger$ & 0.75 \\
\hline
\end{tabular}

Nota.. Mnd = mediana; $I I Q=$ intervalo interquartil; $S E=$ erro padrão; $U=$ Teste de Mann-Whitney

$* * p<.01 ; * p<.05$

$\dagger p$-valores corrigidos pela taxa de falsa descoberta (false discovery rate - FDT) 


\section{Discussão}

Esse estudo comparou indicadores do FAA e habilidades comunicativo-pragmáticas entre pais e mães de crianças com TEA e pais e mães de crianças com DT. Foi verificado que os dois grupos de pais apresentaram escores abaixo dos pontos de corte nas três dimensões do BAPQ, contrariando a hipótese de que o grupo TEA apresentaria escores que correspondem à presença de traços autísticos subclínicos ( $\left.\mathrm{FAA}^{+}\right)$. Apesar disso, houve diferenças significativas entre os grupos nos indicadores de FAA + , com desempenho inferior dos pais e mães de crianças com TEA nas dimensões de Linguagem Pragmática e Escore Total do BAPQ. Além disso, os pais de crianças com TEA também obtiveram escores mais baixos em QI verbal, QI total e habilidades comunicativo-pragmáticas em comparação aos pais de crianças sem TEA.

Esses resultados estão alinhados com estudos que encontraram subgrupos de traços autísticos subclínicos entre parentes adultos de indivíduos com TEA para definir limites e prevalência do FAA (Bora, Aydin, Saraç, Kadak \& Köse, 2017; Rubenstein \& Chawla, 2018; Sasson, Lam, Parlier, Daniels \& Piven, 2013; Wheelwright et al., 2010). Vários desses estudos categorizaram esses pais em FAA e grupos não afetados com base em pontuações de corte arbitrárias de medidas desse construto (Losh et al., 2008; Piven \& Palmer, 1999; Sasson et al., 2013). Sasson et al. (2013) relatou que entre 14 a $23 \%$ dos pais de pessoas com TEA e entre 5 e $9 \%$ dos pais do grupo comparativo apresentavam traços subclínicos que caracterizavam o FAA. A revisão realizada por Rubenstein e Chawla (2018) demonstrou que a porcentagem de pais de crianças com autismo que apresentavam traços autísticos, caracterizando o FAA, variou de 2.6 a $80 \%$. No presente estudo, a maior dificuldade na dimensão Linguagem Pragmática dos pais de crianças com TEA sugere a presença de traços subclínicos.

Não somente as habilidades comunicativo-pragmáticas apresentam-se como possíveis marcadores da presença de traços autísticos. Quanto ao funcionamento social, o estudo de De Groot e Van Strien (2017) encontrou que ele varia ao longo de um continuum de traços autísticos na população geral e apoiam a noção de que diferenças individuais estão relacionadas ao FAA. As análises de regressão demonstraram que características de autismo na população com desenvolvimento típico predizem a frequência com que indivíduos engajam em diferentes comportamentos sociais e o nível de desconforto que experimentam nessas situações. Ainda, as análises de correlação mostraram que todos os comportamentos sociais investigados estiveram relacionados a um ou mais traços autísticos específicos. Esses dados, juntamente com outros que vem sendo reportados mais recentemente na literatura (para revisão, ver Cribb et al., 2016) fortalecem a noção de que traços subclínicos relacionados ou que se assemelham aos encontrados no TEA possivelmente estão presentes na população em geral.

Wheelwright et al. (2010) dividiram o FAA em três níveis diferentes, com base na gravidade dos escores do Autism Quotient (AQ), uma medida de FAA administrada a genitores de crianças com TEA, incluindo classificações que denominaram como: FAA ( 1 a 2 desvios padrão acima da média no $\mathrm{AQ}$ ); fenótipo médio de autismo (indivíduos que classificam entre 2 e 3 desvios padrão acima da média) e fenótipo de autismo limítrofe, (tradução livre, indivíduos com desvios padrão $>3$, em média). Neste estudo, os pais do grupo TEA apresentaram escores maiores nos três níveis em relação aos pais do grupo comparativo (Wheelwright et al., 2010). Ruta et al. (2012) também encontraram diferenças no AQ entre pais de indivíduos com e sem TEA, em que $13.5 \%$ dos pais do grupo TEA e $1 \%$ de pais do grupo comparativo apresentavam fenótipo médio de autismo ou fenótipo de autismo limítrofe. Porém, não houve diferença significativa na proporção de indivíduos que classificaram 1 a 2 desvios padrão acima da média do AQ entre os grupos, ou seja, que configuraria FAA. Esses resultados são coerentes com os do presente estudo à medida em que não houve 
diferença entre os grupos em todas as medidas de FAA utilizadas separadamente. Entretanto, dificuldades na linguagem pragmática, destacou-se como um possível traço autístico subclínico.

Quanto às diferenças encontradas em habilidades comunicativo-pragmáticas, avaliadas por meio da Bateria MAC, o desempenho inferior do grupo clínico foi nas habilidades de Discurso conversacional, Prosódia emocional - Compreensão e Interpretação de Metáforas e de Atos de Fala Indiretos. Esses dados apresentam concordância com outros estudos ao confirmarem a presença de maiores dificuldades quanto ao domínio sociocomunicativo em parentes de primeiro grau de pessoas com TEA. Por exemplo, Sucksmith et al. (2011) realizaram uma revisão acerca de pesquisas que investigaram os traços fenotípicos que delineiam o FAA. Dentre tantos campos analisados, foi concluído que as dificuldades no uso da linguagem para propósitos comunicativos e sociais (pragmática) são componentes do FAA (Bernier et al., 2012; Gerdts et al., 2013; Sucksmith et al., 2011). No nível comportamental, os principais estudos investigam os aspectos pragmático-semânticos, atrasos de linguagem e comunicação. Já no nível cognitivo, o foco das investigações tem sido sobre as habilidades de linguagem receptiva e expressiva. No entanto, os resultados, em geral, de estudos que analisam o desempenho da linguagem no nível cognitivo não fundamentam tão fortemente a inclusão dessas características no FAA quanto os que focam nas habilidades comunicativo-pragmáticas (Sucksmith et al., 2011).

Tendo em vista as inconsistências reportadas na literatura sobre a ocorrência de dificuldades nos aspectos estruturais da linguagem, o desempenho mais baixo do grupo clínico em relação ao grupo comparativo no presente estudo sugere uma possível relação entre as variáveis QI (especialmente o QI verbal) e FAA. De fato, o estudo de Bishop, Maybery, Wong, Maley e Hallmayer (2006) demonstrou que crianças com autismo, cujos escores na Children's Communication Checklist - 2 (CCC-2) confirmaram a presença de dificuldades em comunicação, também apresentaram baixo QI verbal. Contudo, não se sabe se o resultado de Bishop et al. (2006) também se estenderia aos familiares das crianças.

Na presente pesquisa, foram encontrados achados expressivos, com tamanhos de efeito moderados, a partir das análises de comparação entre os grupos, confirmando a sensibilidade da Bateria MAC para identificar fatores do processamento da comunicação no campo do TEA. Portanto, pode-se considerar que ela se mostra como uma possibilidade de medida neuropsicológica para a identificação de endofenótipos do autismo. Esses instrumentos, que são bem consolidados internacionalmente, permitiram a realização da avaliação direta dos participantes, juntamente com a WASI, e outros questionários e escalas (BAPQ) para caracterização de dados sociodemográficos e questões no âmbito emocional, diferentemente de alguns estudos que empregaram somente escalas e questionários.

Quanto à caracterização da amostra, os grupos foram inicialmente equiparados por idade e sexo, porém foram observadas diferenças em relação ao NSE. Por um lado, a escolha pelo método de amostragem bola de neve (que inicialmente poderia contribuir para uma melhor equiparação entre os grupos), mostrou-se um fator que dificultou o controle de variáveis visando a equiparação dos participantes porque os grupos clínico e comparativo pertencem a níveis socioeconômicos diferentes. Por outro lado, foi possível verificar que o NSE não se relacionou com as variáveis cognitivo-linguísticas dentro de cada grupo, o que sugere que esse fator não interferiu nos resultados de comparação, nos quais foram encontradas diferenças entre moderadas a fortes nos domínios avaliados. Apesar disso, é importante considerar que o NSE pode interagir com fatores neuropsicológicos (Hackman, Farah \& Meaney, 2010; Piccolo, Sbicigo, Grassi-Oliveira \& Salles, 2014).

Por fim, algumas limitações desse estudo foram o número reduzido de participantes, o que pode ter dificultado resultados mais expressivos. Além disso, o procedimento de correção dos instrumentos não foi 
"às cegas", o que pode ter sido uma fonte de viés. Outros estudos também reportaram dificuldades quanto ao número amostral e vieses metodológicos, incluindo as variáveis QI e FAA (ex.: Cribb et al., 2016). No entanto, na realidade brasileira, entre outros fatores, cadastros nacionais com bancos de dados dessa população (TEA) são escassos e a interlocução entre os centros de pesquisa ainda não é expressiva, fazendo com que os pesquisadores lancem mão de alternativas para recrutarem seus participantes. Portanto, estudos nacionais que possam replicar os achados preliminares da presente pesquisa, porém com processos de seleção de amostras aleatórios e maior tamanho amostral, poderão contribuir para a confirmação ou não dos resultados encontrados. Em contrapartida, frente a esses aspectos, também se pode questionar se traços subclínicos de autismo podem estar espalhados na população em geral, como já apontado por alguns pesquisadores (Camodeca et al., 2018; Cribb et al., 2016; De Groot \& Van Strien, 2017).

\section{Referências}

American Psychiatric Association. (2013). Diagnostic and statistical manual of mental disorders. Washington, D.C.: Autor.

Associação Brasileira de Empresas de Pesquisa (2015). Critério de Classificação Econômica Brasil - CCEB - Dados com base no levantamento socioeconômico 2015. Recuperado de $\mathrm{http} / / \mathrm{www}$.abep.org

Baron-Cohen, S., Wheelwright, S., Skinner, R., Martin, J., \& Clubley, E. (2001). The Autism Spectrum Quotient (AQ): Evidence from Asperger syndrome/high-functioning autism, males and females, scientists and mathematicians. Journal of Autism and Developmental Disorders, 31(1), 5-17. https://doi.org/0162-3257/01/0200$0005 \$ 19.50 / 0$

Benjamini Y, \& Hochberg Y. (1995). Controlling the false discovery rate: A practical and powerful approach to multiple testing. Journal of the Royal Statistical Society: Series B (Statistical Methodology),57, 289-300.

Bernier, R., Gerdts, J., Munson, J., Dawson, G., \& Estes, A. (2012). Evidence for broader autism phenotype characteristics in parents from multiple-incidence autism families. Autism Research, 5(1), 13-20. https://doi.org/10.1002/aur.226

Bishop, D. V. M., Maybery, M., Wong, D., Maley, A., \& Hallmayer, J. (2006). Characteristics of the broader phenotype in autism: A study of siblings using the children's communication checklist-2. American Journal of Medical Genetics, 141B(2), 117-122. https://doi.org/10.1002/ ajmg.b.30267

Bora, E., Aydın, A., Saraç, T., Kadak, M. T., \& Köse, S. (2017). Heterogeneity of subclinical autistic traits among parents of children with autism spectrum disorder: Identifying the broader autism phenotype with a data-driven method. Autism Research, 10, 321-326. https:// doi.org/10.1002/aur.1661

Camodeca, A., Todd, K. Q., \& Hosack, A. (2018). Intact verbal fluency abilities in the Broad Autism Phenotype. Psychiatry research, 270, 443-452. https://doi.org/10.1016/j.psychres.2018.10.006

Camodeca, A., \& Voelker, S. (2016). Automatic and controlled processing and the Broad Autism Phenotype. Psychiatry research, 235, 169-176. https://doi.org/10.1016/j.psychres.2015.11.012

Chuthapisith, J., Ruangdaraganon, N., Sombuntham, T., \& Roongpraiwan, R. (2007). Language development among the siblings of children with autistic spectrum disorder. Autism, 11(2), 149-160. https://doi.org/10.1177/1362361307075706

Cohen, J. (1988). Statistical power analysis for the behavioral sciences. Hillsdale, NJ: Lawrence Erlbaum Associates Publishers.

Cribb, S. J., Olaithe, M., Di Lorenzo, R., Dunlop, P. D., \& Maybery, M. T. (2016). Embedded figures test performance in the broader autism phenotype: A meta-analysis. Journal of Autism 
and Developmental Disorders, 46, 2924- 2939. https://doi.org/10.1007/s10803-016-2832-3

Cruz, L. L. P. (2012). Ocorrência de fenótipo ampliado do autismo em pais de crianças portadoras $d a$ síndrome (Dissertação de mestrado, Instituto de Previdência dos Servidores do Estado de Minas Gerais, Minas Gerais, Brasil).

Davidson, J., Goin-Kochel, R. P., Green-Snyder, L. A., Hundley, R. J., Warren, Z., \& Peters, S. U. (2014). Expression of the broad autism phenotype in simplex autism families from the simons simplex collection. Journal of autism and developmental disorders, 44, 1-8. https://doi. org/10.1007/s10803-012-1492-1

Dawson, G., Webb, S., Schellenberg, G. D., Dager, S., Friedman, S., Aylward, \& Richards, T. (2002). Defining the broader phenotype of autism: Genetic, brain, and behavioral perspectives. Development and Psychopathology, 14(3), 581-611. https://doi.org/10.1017/S0954579402003103

De Groot, K., \& Van Strien, J. W. (2017). Evidence for a broad autism phenotype. Advances in Neurodevelopmental Disorders, 1(3), 129-140. https://doi.org/10.1007/s41252-017-0021-9

Endres, R. G., Lampert, S. S., Schuch, J. B., Roman, T., \& Bosa, C. A. (2015). The broader autism phenotype in parents of children with autism spectrum disorder - ASD. Psicologia: Teoria e Pesquisa, 31(3), 285-292. https://doi. org/10.1590/0102-37722015032268285292

Ferré, P., Lamelin, F., Côté, H., Ska, B., \& Joanette, Y. (2011). Protocole MEC-P (Protocole Montréald'Évaluation de la Communication (version de Poche)). Isbergues (France): Ortho Édition.

Flax J., Gwin, C., Wilson, S. Fradkin, Y., Buyske, S., \& Brzustowicz, L. (2019). Social (pragmatic) communication disorder: Another name for the Broad Autism Phenotype? Autism, 23. 1-11. https://doi.org/10.1177/1362361318822503

Fleitlich, B., Cortázar, P. G., \& Goodman, R. (2000). Questionário de capacidades e dificuldades (SDQ). Infanto-Revista de Neuropsiquiatria da Infância e Adolescência, 8(1), 44-50.
Folstein, S. E., Santangelo, S. L., Gilman, S. E., Piven, J., Landa, R., Lainhart J., ..., Wzorek M. (1999). Predictors of cognitive test patterns in autism families. The Journal of Child Psychology and Psychiatry and Allied Disciplines, 40(7), 11171128.

Fombonne, E., Bolton, P., Prior, J., Jordan, H., \& Rutter, M. (1997). A family study of autism: Cognitive patterns and levels in parents and siblings. Journal of Child Psychology and Psychiatry, 38(6), 667-683.

Fonseca, R. P., Parente, M. A. M. P., Côté, H., Ska, B., \& Joanette, Y. (2008). Apresentando um instrumento de avaliação da comunicação à fonoaudiologia brasileira: bateria MAC. Pró Fono Revista de Atualização Científica, 20(4), 285-92.

Fritz, C. O., Morris, P. E., \& Richler, J. J. (2012). Effect size estimates: Current use, calculations, and interpretation. Journal of Experimental Psychology: General, 141(1), 2-18. https://doi. org/10.1037/a0024338

Gerdts, J. A., Bernier, R., Dawson, G., \& Estes, A. (2013). The broader autism phenotype in simplex and multiplex families. Journal of Autism and Developmental Disorders, 43(7), 1597-1605. https://doi.org/10.1007/s10803012-1706-6

Hackman, D. A., Farah, M. J., \& Meaney, M. J. (2010). Socioeconomic status and the brain: Mechanistic insights from human and animal research. Nature Reviews. Neuroscience, 11(9), 651-659. https://doi.org/10.1038/nrn2897

Hallmayer, J., Cleveland, S., Torres, A., Phillips, J., Cohen, B., Torigoe, T., ..., Risch, N. (2011). Genetic heritability and shared environmental factors among twin pairs with autism. Archives of General Psychiatry,68, 1095-1102. https:// doi.org/10.1001/archgenpsychiatry.2011.76

Harding, T. W., De Arango, M. V., Baltazar, J., Climent, C. E., Ibrahim, H. H. A., Ladrido-Ignacio, ..., Wig, N. N. (1980). Mental disorders in primary health care: A study of their frequency 
and diagnosis in four developing countries. Psychological Medicine, 10, 231-241.

Hurley, R. S. E., Losh, M., Parlier, M., Reznick, J. S., \& Piven, J. (2007). The broad autism phenotype questionnaire. Journal of Autism and Developmental Disorders, 37(9),1679-90. https://doi. org/10.1007/s10803-006-0299-3

Ingersoll, B. (2010). Broader autism phenotype and nonverbal sensitivity: Evidence for an association in the general population. Journal of Autism Developmental Disorders, 40, 590-598. https:// doi.org/10.1007/s10803-009-0907-0

Lee, M., Naya, K., Maltman, N., Hamburger, D., Martin, G. E., Gordon, P. C., \& Losh, M. (2019). Understanding social communication differences in Autism Spectrum Disorder and first-degree relatives: A study of looking and speaking. Journal of Autism and Developmental Disorders, 1-14. https://doi.org/10.1007/ s10803-019-03969-3

Lindgren, K. A., Folstein, S. E., Tomblin, J. B., \& Tager-Flusberg, H. (2009). Language and reading abilities of children with autism spectrum disorders and specific language impairment and their first-degree relatives. Autism Research, 2 , 22-38. https://doi.org/10.1002/aur.63

Losh, M., Childress, D., Lam, K., \& Piven, J. (2008). Defining key features of the broad autism phenotype: A comparison across parents of multipleand single-incidence autism families. American Journal of Medical Genetics, 147B, 424-433. https://doi.org/10.1002/ajmg.b.30612

Mari, J. J., \& Williams, P. (1986). A validity study of a psychiatric screening questionnaire (SRQ$20)$ in primary care in the city of São Paulo. The British Journal of Psychiatry, 148, 23-26. https://doi.org/10.1192/bjp.148.1.23

Mottron, L., Bouvet, L., Bonnel, A., Samson, F., Burack, J. A., Dawson, M., \& Heaton, P. (2013). Veridical mapping in the development of exceptional autistic abilities. Neuroscience \& Biobehavioral Reviews, 37(2), 209-28. https://doi. org/10.1016/j.neubiorev.2012.11.016
Naschmias, C., \& Naschmias, D. (1996). Research methods in the social sciences. London: Arnold.

Piccolo, L. R., Sbicigo, J. S., Grassi-Oliveira, R., Salles, J. F. (2014). Do SES and stress reactivity impact neurocognitive performance? Psychology \& Neuroscience, 7(4), 567-575. https://doi. org/10.3922/j.psns.2014.4.16

Pilowsky, T., Yirmiya, N., Gross-Tsur, V., \& Shalev, R. S. (2007). Neuropsychological functioning of siblings of children with autism, siblings of children with developmental language delay, and siblings of children with mental retardation of unknown genetic etiology. Journal of Autism and Developmental Disorders, 37, 537-552. https://doi.org/10.1007/s10803-006-0185-z

Pilowsky, T., Yirmiya, N., Shalev, R. S., \& GrossTsur, V. (2003). Language abilities of siblings of children with autism. Journal of Child Psychology \& Psychiatry, 44, 914-925. https://doi. org/10.1111/1469-7610.00175

Piven, J. (2001). The broad autism phenotype: A complementary strategy for molecular genetic studies of autism. American Journal of Medical Genetics, 105(1), 34-35. https://doi.org/10.1002/10968628(20010108)105:1<34:AID-AJMG1052> 3.0.CO;2-D

Piven, J., \& Palmer, P. (1997). Cognitive deficits in parents from multiple-incidence autism families. Journal of Child Psychology and Psychiatry, 38, 10111021. https://doi.org/10.1111/j.1469-7610.1997. tb01618.x

Piven, J., \& Palmer, P. (1999). Psychiatric disorder and the broad autism phenotype: Evidence from a family study of multiple-incidence autism families. American Journal of Psychiatry, 156(4), 557-63. https://doi.org/10.1176/ ajp.156.4.557

Piven J., Palmer P., Landa R., Santangelo S., Jacobi D., \& Childress, D. (1997). Personality and language characteristics in parents from multiple-incidence autism families. Neuropsychiatric Genetics, 74, 398-411. Recuperado de https:// www.ncbi.nlm.nih.gov/pubmed/9259376 
Rubenstein, E., \& Chawla, D. (2018). Broader autism phenotype in parents of children with autism: A systematic review of percentage estimates. Journal of child and family studies, 27, 17051720. https://doi.org/10.1007/s10826-0181026-3

Ruparelia, K., Manji, K., Abubakar, A., \& Newton, C. R. (2017). Investigating the evidence of behavioral, cognitive, and psychiatric endophenotypes in autism: A Systematic review. Autism Research and Treatment, 2017. https:// doi.org/10.1155/2017/6346912

Ruta, L., Mazzone, D., Mazzone, L., Wheelwright, S., \& Baron-Cohen, S. (2012). The autism-spectrum quotient- Italian version: A cross-cultural confirmation of the broader autism phenotype. Journal of Autism and Developmental Disorders, 42(4), 625-33. https://doi.org/10.1007/ s10803-011-1290-1

Sasson, N. J., Lam, K. S., Parlier, M., Daniels, J. L., \& Piven, J. (2013). Autism and the broad autism phenotype: Familial patterns and intergenerational transmission. Journal of Neurodevelopmental Disorders, 5(1), 11. https://doi. org/10.1186/1866-1955-5-11

Smalley, S. L., \& Asarnow, R. F. (1990). Brief report: Cognitive subclinical markers in autism. Journal of Autism and Developmental Disorders, 20, 271-278. https://doi.org/10.1007/BF02284724

Sucksmith, E., Roth, I., \& Hoekstra, R. A. (2011). Autistic traits below the clinical threshold: Re-examining the broader autism phenotype in the $21^{\text {st }}$ century. Neuropsycholy Review, 21, 360-389. https://doi.org/10.1007/s11065-0119183-9

Taylor, L. J., Maybery, M. T., Wray, J., Ravine, D., Hunt, A., \& Whitehouse, A. J. (2013). Brief report: Do the nature of communication im- pairments in autism spectrum disorders relate to the broader autism phenotype in parents? Journal of Autism and Developmental Disorders,43(12), 2984-2989. https://doi.org/10.1007/ s10803-013-1838-3

The Psychological Corporation. (1999). Wechsler Abbreviated Scale of Intelligence Manual. San Antonio: APA.

Trentini, C. M., Yates, D. B., \& Heck, V. S. (2014). Escala de inteligência Wechsler abreviada (WASI): manual profissional. São Paulo: Pearson.

Tick, B., Bolton, P., Happé, F., Rutter, M., \& Rijsdijk, F. (2016). Heritability of autism spectrum disorders: A meta-analysis of twin studies. Journal of Child Psychology and Psychiatry, 57(5), 585-595. https://doi.org/10.1111/jcpp.12499

Van der Hallen, R., Evers, K., Brewaeys, K., Van den Noortgate, W., \& Wagemans, J. (2015). Global processing takes time: A meta-analysis on local-global visual processing in ASD. Psychological Bulletin, 141(3), 549-573. https:// doi.org/10.1037/bul0000004

Waye, M. M., \& Cheng, H. Y. (2018). Genetics and epigenetics of autism: A review. Psychiatry and clinical neurosciences, 72(4), 228-244. http:// doi.org/10.1111/pen.12606

Wheelwright, S., Auyeung, B., Allison, C., \& Baron-Cohen, S. (2010). Defining the broader, medium and narrow autism phenotype among parents using the Autism Spectrum Quotient (AQ). Molecular Autism, 1, 1-10. https://doi. org/10.1186/2040-2392-1-10

Whitehouse, A. J. O., Barry, J. G., \& Bishop, D. V. M. (2007). The broader language phenotype of autism: A comparison with specific language impairment. Journal of Child Psychology and Psychiatry, 48(8), 822-830. https://doi. org/10.1111/j.1469-7610.2007.01765.x 